\title{
Déterminants socio-économiques de l'adoption des pratiques culturales de gestion de la fertilité des sols utilisées dans la commune de Ouaké au Nord- Ouest du Bénin
}

\author{
Jacob Afouda YABI ${ }^{1 *}$, François Xavier BACHABI ${ }^{2}$, Innocent Adédédji LABIYI ${ }^{1}$, \\ Christian Agbatchi ODE ${ }^{1}$ et Romaine Lalé. AYENA ${ }^{1}$ \\ ${ }^{1}$ Laboratoire d'Analyse et de Recherche sur les Dynamiques Economique et Sociale (LARDES), Faculté \\ d'Agronomie, Université de Parakou, Bénin. \\ ${ }^{2}$ Département de Production Végétale (DPV), Ecole Nationale des Sciences et Techniques Agricoles de \\ Djougou (ENSTA-Dj), Université de Parakou, Bénin. \\ "Corresponding author, E-mail: ja_yabi@yahoo.com; Tél: +22997320856
}

\section{RESUME}

L'objectif de ce travail a été d'identifier les facteurs qui influencent l'adoption des pratiques culturales de gestion de la fertilité des sols utilisées dans la commune de Ouaké. Cent vingt (120) chefs d'exploitation agricoles ont été échantillonnés puis enquêtés de façon aléatoire et simple. Les données ainsi collectées ont été analysées à l'aide d'une régression logistique. Les résultats révèlent que le sexe, le mode de faire-valoir des terres mises en culture, l'appartenance à un groupement et l'accès à l'engrais minéral ont une influence positive sur l'adoption de la fumure minérale tandis que pour la pratique d'agroforesterie, les variables taille du ménage et mode de faire-valoir la déterminent avec une influence positive, mais les variables nombre d'actifs agricoles et l'appartenance à un groupement ont un impact négatif sur la pratique d'agroforesterie. Quant à la pratique de lutte antiérosive ; le sexe, l'âge, l'appartenance à un groupement, et l'accès à l'engrais minéral influencent négativement son adoption tandis que le niveau d'instruction, la taille du ménage et le statut social du producteur déterminaient positivement l'adoption de cette pratique. Ainsi, la pratique traditionnelle qu'est la rotation et l'association de cultures appropriées est déterminée positivement par l'âge du producteur. Enfin, le niveau d'instruction et le nombre d'actifs agricoles influencent négativement l'adoption de cette pratique culturale. Ces résultats suggèrent aux décideurs des politiques agricoles de prendre en compte les différents facteurs d'influence significative dans la promotion de la conservation des terres agricoles.

(c) 2016 International Formulae Group. All rights reserved.

Mots clés : Pratiques culturales, fertilité de sols, régression logistique, conservation des sols, Commune de Ouaké.

\section{Socio-economic determinants of the adoption of agricultural practices of soil fertility management in North-Western Benin: Case study of Ouaké municipality}




\begin{abstract}
The objective of this work was to identify the factors that influence the adoption of agricultural practices of soil fertility management used in the municipality of Ouaké. One hundred and twenty (120) agricultural producers were randomly sampled and surveyed. The collected data were analyzed using logistic regressions. The results revealed that sex, land tenure, belonging to a group and access to mineral fertilizer had a positive influence on the adoption of the mineral fertilizer while for agroforestry practice, the variables household size and land tenure had a positive impact on the adoption of this practice, but the variables number of farm assets and belonging to a group, have a negative impact on agroforestry. As for the erosion control practice; sex, age, membership in a group, and access to mineral fertilizer negatively influenced its adoption while the level of education, household size and richness status of the producer determined positively this practice adoption. Finally, the traditional practice known as appropriate crops rotation and association was positively determined by age. Thus, the level of education and the number of farm assets influenced negatively this cultural practices adoption. These results suggest agricultural policy makers to take into account these different factors of significant influence in promoting the sustainable conservation of agricultural land fertility. (C) 2016 International Formulae Group. All rights reserved.
\end{abstract}

Keywords: Cultural practices, soil fertility, logistic regression, soil conservation, Ouaké municipality.

\section{INTRODUCTION}

En Afrique de l'Ouest, précisément dans la zone de l'Union Economique Monétaire Ouest Africaine (UEMOA), l'agriculture contribue pour $30 \%$ au PIB et emploie plus de $50 \%$ de la population active (Renard et al., 2004). Ainsi, pour Jama et Gonzalo (2008), le développement agricole se trouve au cœur des stratégies de réduction de la pauvreté et de la sécurité alimentaire dans la plupart des pays en voie de développement. Cependant, cette agriculture se trouve confrontée dans la plupart de ces pays à plusieurs contraintes dont l'une des plus importantes est le phénomène de la dégradation des sols qui limite le potentiel de l'agriculture (Serme et al., 2015). L'acuité de ce problème au Bénin varie d'une localité à une autre du territoire national. $\mathrm{Au}$ NordBénin, les résultats ont montré un abandon de la jachère qui était la technique de restauration des sols (Batamoussi Hermann et al., 2015). Ces constats sont plus accentués dans la commune de Ouaké où les terres cultivables sont sujettes à l'érosion hydrique, éolienne et occupées en grande partie par la cuirasse latéritique.

Plusieurs stratégies ont été développées par les producteurs pour réduire la baisse de la fertilité des sols et accroître leurs revenus en vue d'une amélioration de leur condition de vie. Ces stratégies combinaient entre autres le travail du sol, la jachère de courte durée, l'utilisation de la fumure organique. D'autres initiatives comme la jachère améliorée ont été introduites par les projets et programmes de protection de l'environnement. Ainsi, une étude exploratoire conduite par Nouatin (2008) a permis d'identifier quatre grands groupes de pratiques de gestion de la fertilité des sols mises en œuvre par les producteurs dans leur système de production dans la zone. Ce sont: la pratique de fumure minérale, les pratiques agro-forestières, la pratique antiérosive et les pratiques de rotations et d'associations de cultures appropriées. Cependant, ces innovations proposées ont été peu adoptées par les producteurs. Il apparaît donc opportun de chercher à comprendre quels sont les facteurs qui déterminent l'adoption de ces pratiques afin d'œuvrer efficacement pour l'acceptation de ses pratiques en milieu rural en vue d'une meilleure gestion de la fertilité des sols. C'est dans la perspective de trouver une approche de solution à cette interrogation que s'inscrit la présente étude. Cette étude vise spécifiquement à identifier les variables qui influencent l'adoption des différentes 
pratiques de gestion et de fertilité des sols dans la commune de Ouaké au Nord-Bénin.

\section{MATERIEL ET METHODES}

Zone d'étude et base de données

La zone d'étude est la commune de Ouaké située au Nord-Ouest du département de la Donga entre les parallèles $9^{\circ}$ et $10^{\circ}$ de latitude Nord et les méridiens $1^{\circ}$ et $2^{\circ}$ de longitude Est. Commune frontalière, elle partage $70 \mathrm{~km}$ de frontière avec la République du Togo. Le relief se compose d'une pénéplaine très ondulée issue de l'érosion d'une vieille surface d'aplanissement et présentant de faibles dénivellations à pente plus ou moins inclinées donnant lieu à de vastes vallées de forme évasée et peu profondes, des collines résiduelles isolées ou non aux versants doux d'altitude modeste par endroits. La commune de Ouaké, située dans une savane arborée et herbacée avec un climat du type soudanien humide, comporte deux (02) saisons : une saison de pluie de mai à octobre et une saison sèche de novembre à avril. La pluviométrie moyenne annuelle calculée sur 20 ans est de $1250 \mathrm{~mm}$. Mais la hauteur de pluie avoisine $1400 \mathrm{~mm}$ par an. La commune de Ouaké dispose d'une gamme variée de sols. On y rencontre des sols latéritiques, caillouteux, sablonneux, sabloargileux et les sols minéraux bruts sur socle cristallin souvent profonds. Ces sols sont fortement dégradés par l'érosion.

L'unité de recherche est l'exploitant agricole c'est-à-dire tout producteur utilisant les pratiques culturales de gestion de la fertilité des sols sur les parcelles mises en valeur. L'échantillonnage a été fait de façon raisonnée et a pris en compte cent vingt (120) producteurs choisis, selon leur disponibilité et l'intensification des pratiques culturales utilisées dans la commune de Ouaké signalée dans les études diagnostiques. Quatre (04) villages (Tchaladè, Alayomdè et Assodè) ont été échantillonnés à raison de quarante (40) exploitants agricoles par village. Trois (03) catégories de cibles: les paysans vulnérables cultivant des céréales sur les terres appauvries, presque dénudées du plateau de Ouaké; les producteurs de riz qui occupent les rares basfonds de Ouaké qui leur permettent de produire à la fois pour la subsistance et le marché ; les femmes productrices de compost, généralement épandu dans les champs de cases.

Le questionnaire individuel a permis d'obtenir des données quantitatives et qualitatives sur les caractéristiques sociodémographiques du producteur, les caractéristiques des différentes pratiques de gestion de la fertilité des sols identifiées dans la commune de Ouaké. Sur la base de ces données primaires, les régressions logistiques ont été estimées afin d'identifier les facteurs influençant chaque pratique culturale. En complément, sur la base d'un questionnaire, des entretiens semi-structurés ont été organisés pour collecter des données qualitatives et quantitatives. Enfin, pour s'assurer de la véracité des informations recueillies, des observations et la triangulation des informations fournies ont été réalisées.

\section{Modèles empirique et conceptuel}

Dans le cadre de cette étude, nous avons retenu le modèle Logit, souvent utilisé dans les études d'adoption des technologies pour des raisons de commodité (Cimmyt, 1993). Aussi ce modèle a eu à faire ses preuves dans plusieurs études d'adoption notamment celles de Adésina et al. (2000) et de Sale et al. (2014). En effet, les modèles Logit polytomiques non ordonnés sont moins intensifs en calculs mais requièrent la non violation de l'hypothèse de l'indépendance des alternatives non pertinentes (Madalla, 1985; Cramer, 1991). Quant aux modèles Probit polytomiques non ordonnés, ils sont plus intensifs en calculs et n'imposent pas de restrictions particulières (Sale et al., 2014). Dans ce travail, nous utilisons le modèle Logit, qui facilite la manipulation des résultats (Hurlin, 2003).

La décision d'adopter une innovation n'intervient que lorsque l'effet combiné des facteurs atteint une valeur à partir de laquelle 
le décideur accepte d'utiliser ou adopter l'innovation. En se mettant dans l'hypothèse que l'effet est mesuré par un indice non observable $\mathrm{I}_{\mathrm{d}}$ pour le décideur $\mathrm{d}$ et $\mathrm{I}_{0 \mathrm{~d}}$ la valeur critique de l'indice à partir de laquelle il adopte la technologie, deux cas de figures peuvent se présenter :

Si $I_{d}$ est supérieur ou égal à $I_{0 d}$, alors il adopte la technologie et la variable d'adoption $Y$ prend la valeur 1. Plus l'indice $I_{d}$ est supérieur à la valeur critique, plus la probabilité pour que le producteur adopte est élevée. $S i I_{d}$ est inférieur à $I_{0 d}$, il rejette l'innovation et $\mathrm{Y}$ est égal à 0 .

En formulation mathématique, il vient que :

$$
\left\{\begin{array}{l}
\mathrm{I}_{\mathrm{d}} \geq \mathrm{I}_{0 \mathrm{~d}} \Rightarrow \mathrm{Y}=1 \\
\mathrm{I}_{\mathrm{d}}<\mathrm{I}_{0 \mathrm{~d}} \Rightarrow \mathrm{Y}=0
\end{array}\right.
$$

Pour l'individu $d$, l'indice $I_{d}$ peut être une combinaison linéaire de variables $\mathrm{Xi}$ qui déterminent l'adoption et de coefficients $\beta \mathrm{i}$ à estimer. Son expression est alors mathématiquement donnée par :

$$
I_{d}=\sum_{i=1}^{k} \beta_{i} X_{i d}
$$

Avec Xid la iième variable indépendante expliquant l'adoption de la technologie par l'individu d et $\beta \mathrm{i}$ son paramètre correspondant à estimer.

La probabilité Pd pour que l'individu d adopte l'innovation est alors :

$$
\mathrm{Pd}=\mathrm{P}(\mathrm{Y}=1)
$$

Comme l'indice $\mathrm{I}_{0} \mathrm{~d}$ est une variable aléatoire, si nous désignons par $\mathrm{F}$ sa fonction de probabilité cumulée ou fonction de répartition, il vient que :

$\left\{\begin{array}{l}P(Y=1)=P\left(I_{0 d} \leq I_{d}\right)=F\left(I_{d}\right) \\ P(Y=0)=1-F\left(I_{d}\right)\end{array}\right.$

La forme fonctionnelle de $\mathrm{F}$ est déterminée par celle de la fonction de densité de probabilité de la variable aléatoire Id. Pour le modèle logit, il s'agit d'une fonction logistique de la forme :

$$
F(x)=\frac{1}{1+e^{-x}}=\frac{1}{1+e^{-(\beta o+\beta i z)}}
$$

\section{Modèle économétrique}

L'équation empirique issue du modèle théorique, se présente comme suit :

$$
P(Y i=1 / A D O P T \quad)=\frac{1}{1+e^{-X}}
$$

Avec

$X=\beta_{0}+\beta_{1} A G E+\beta_{2} E D U C+\beta_{3} T A I L M+\beta_{4}$ APPGROP $+\beta_{5}$ NACT $+\beta_{6}$ STAT+ $\beta_{7} A C C I N T+\beta_{8}$ CONTACT $+\beta_{9} S E X+\beta_{10}$ $M F V+e_{i} \quad$ (6)

Où $\beta_{0}$ est le terme constant ; $\beta \mathrm{i}$ les coefficients à estimer, et $\mathrm{e}_{\mathrm{i}}$ les termes d'erreur. Plusieurs techniques sont utilisées pour juger de la qualité du modèle : elle peut être donnée par la vraisemblance du modèle qui suit une loi de Chi-deux. Le modèle est dit globalement bon, lorsque la valeur de la vraisemblance est supérieure à celle du Chideux au même degré de liberté à un seuil donné $(1 \%, 5 \%$ ou $10 \%)$, ou directement lorsque la probabilité de LR est inférieure au seuil de signification choisi. Les signes indiquent dans quel sens la variation de la variable explicative influe sur la variation de la variable expliquée. A chaque signe des coefficients, est associée une signification qui revêt une grande importance. Leur signification est donnée par une probabilité qui indique dans quel intervalle de confiance on peut compter sur le signe. Les variables marquées $*$ sont significatives à $10 \%$; ** significatives à $5 \%$ et $* * *$ significatives à $1 \%$. Toutes les variables explicatives introduites dans le modèle sont décrites dans le Tableau 1.

Les logiciels SPSS et STATA ont été utilisés respectivement pour les statistiques descriptives et les régressions économétriques. 
L'utilisation des différentes pratiques (fumure minérale, l'agroforesterie, l'association rotation et la lutte antiérosive) pour s'adapter à la baisse de la fertilité des sols: Ces variables sont les différentes variables dépendantes qui sont influencées par des facteurs socio-économiques.

Cymmit (1993), Houndekon et Gogan (1996), distinguent des groupes de facteurs qui sont susceptibles d'influencer l'adoption d'une pratique culturale. Il s'agit des :

Facteurs propres aux producteurs: regroupant le niveau d'éducation de l'exploitant, son expérience en agriculture, son âge, son genre, son niveau de richesse, la taille de son exploitation et le statut social du producteur.

En effet, l'âge constitue aussi un proxy de l'expérience. Les producteurs moins expérimentés sont jeunes. Les jeunes producteurs sont enclins à prendre plus de risque que les plus âgés. Les hommes ont plus d'accès à la terre que les femmes et à l'information sur la disponibilité des intrants. Selon Feder (1982), le nombre d'années d'expérience du producteur devrait influencer négativement l'adoption des technologies; donc l'adoption de nouvelles technologies exige un certain niveau de risque associé à la décision du choix des technologies. Aussi, les producteurs éduqués ont de meilleures aptitudes à appliquer et à diffuser les instructions des services de vulgarisation. La taille du ménage est, dans la littérature, souvent mentionnée comme une variable essentielle dans l'adoption de nouvelles technologies. Elle est positivement liée à l'adoption des innovations.

Facteurs institutionnels : regroupant l'accès au crédit, la tenure foncière, la disponibilité et l'accessibilité d'intrant, la disponibilité des agents de vulgarisation.

Pour Sale et al. (2014), l'accès au crédit est déterminant pour le choix d'une stratégie, car, les producteurs ayant un accès facile aux crédits auront tendance à mieux adopter les nouvelles technologies. De même, Ouédraogo et al. (2010) et Mbétid-Bessane (2014) ont montré l'importance du crédit comme un des déterminants potentiel du comportement des producteurs face à l'innovation.

L'accès à la terre, à l'engrais et le mode de faire valoir sont déterminants pour l'utilisation de différentes pratiques de conservation et de la fertilité des sols dans l'exploitation, car, les producteurs ayant un accès facile aux ressources d'exploitation auront tendance à les adopter.

Enfin l'accès aux services de vulgarisation est un élément important dans les processus d'adoption et de diffusion des innovations. Le contact avec la vulgarisation facilite l'accès à l'information et favorise l'adoption des innovations (Folefack et al., 2012). L'encadrement technique des agriculteurs par les services de vulgarisation influence positivement l'adoption de quelques pratiques cultures de conservation et de fertilité de sols.

\section{RESULTATS \\ Caractéristiques socio-démographiques des exploitations}

Les unités enquêtées au cours de cette étude sont constituées aussi bien d'hommes que de femmes. Les chefs d'exploitation sont en général des hommes $(92 \%)$ sauf des cas rares où la femme représente le chef du ménage $(8 \%)$ par suite du décès de son époux ou d'un divorce. L'âge des producteurs utilisant les pratiques culturales de gestion de la fertilité des sols varie de 24 ans à 59 ans avec une moyenne de 41,74 $\pm 9,19$ ans. Les producteurs utilisant les pratiques de gestion de la fertilité des sols sont des actifs agricoles c'est-à-dire des personnes en âge de travailler dans les champs (tranche d'âge de14 à 60 ans). La majorité des ménages dans l'ensemble de la zone d'étude sont polygames avec une taille variant de 4 à 31 et une moyenne de 11,29 $\pm 5,23$ personnes. Le nombre moyen d'actifs agricoles au sein des ménages est de 5, $30 \pm 2,60$ personnes. Quant au nombre d'inactifs, elle est en moyenne de $6,07 \pm 3,77$ personnes. Le taux de dépendance (rapport entre le nombre d'actifs et le nombre 
d'inactifs) est de ce fait de 1,14 $\pm 0,69$ personnes en moyenne. Seulement $34 \%$ des enquêtés sont scolarisés dont $23,34 \%$ ont un niveau primaire et $10,66 \%$ un niveau secondaire. Aussi seulement 19\% des producteurs sont alphabétisés. L'agriculture constitue la principale activité des producteurs enquêtés (92\% des enquêtés). Plus de troiscinquième (3/5) des enquêtés disposent d'une activité secondaire. Ces activités secondaires sont entre autres l'élevage, le petit commerce, la transformation des produits agricoles pour les femmes, les petits métiers (couture, mécanique, maçonnerie, menuiserie).

Seulement $6 \%$ des enquêtés ont accès au crédit agricole formel octroyé par les structures de la place. Sur la plupart des superficies emblavées, les pratiques culturales de gestion de la fertilité des sols sont utilisées par les producteurs en des proportions différentes. Les producteurs ont donc une préférence en ce qui concerne l'adoption de chacune de ces pratiques (Tableau 2). Ceci renforce la nécessité de s'intéresser aux facteurs déterminants l'adoption de chacune des pratiques que sont la fumure minérale, l'agroforesterie, la lutte antiérosive, la rotation et l'association de cultures appropriées.

Facteurs déterminant l'adoption des pratiques culturales de gestion de la fertilité des sols

L'analyse du Tableau 2 révèle que les modèles d'estimation des facteurs d'adoption des pratiques de fumure minérale, d'agroforesterie et de lutte antiérosive sont significatifs à $1 \%$. Quant au modèle d'estimation des facteurs d'adoption de la rotation et d'association de cultures appropriées, il est significatif à $5 \%$. L'estimation des modèles donne respectivement un pseudo- $\mathrm{R}^{2}$ de McFadden de 0,$34 ; 0,40 ; 0,44 ; 0,193$ pour les modèles d'estimation des facteurs d'adoption des pratiques de fumure minérale, d'agroforesterie, de lutte antiérosive et de rotation et association de cultures appropriées. Les variations des variables explicatives incluses dans le modèle expliquent donc respectivement à $34 \%, 40 \%, 44 \%$ et $19,3 \%$ les variations de l'adoption des pratiques de fumure minérale, d'agroforesterie, de lutte antiérosive et de rotation et association de cultures appropriées. Ces modèles prédisent les résultats obtenus respectivement à $71 \%$, $72 \%, 79 \%$ et $65 \%$.

\section{Déterminants de l'adoption de la pratique de fumure minérale}

Les résultats du modèle logistique identifient quatre facteurs déterminants l'adoption de la pratique de fumure minérale. Le sexe, l'appartenance à un groupement, l'accès à l'engrais minéral et le mode de fairevaloir influencent positivement l'adoption de la pratique de fumure minérale respectivement et significativement au seuil de $10 \%$ (sexe et appartenance à un groupement) et au seuil de 5\% (l'accès à l'engrais minéral et le mode de faire-valoir) (Tableau 3).

Le sexe du producteur (SEX): ces résultats présagent que les hommes utilisent plus cette pratique que les femmes. Dans la zone d'étude, les femmes n'ont pas un accès facile à l'engrais minéral. Elles ne sont généralement pas prises en compte dans le processus d'incitation à la production agricole par l'octroi à crédit de l'engrais minéral. Les femmes qui utilisent la fumure minérale passent généralement par leurs maris.

Le mode de faire-valoir $(M F V)$ : les producteurs ayant hérité les terres les mettent en culture que ceux ayant acquis leurs terres par don. Aussi, très peu de producteurs ayant hérité les terres mises en cultures pensent-ils au devenir des terres exploitées. En effet, ces derniers ne sont pas trop contraints à rester sur les mêmes parcelles au fil des années étant donné la disponibilité en terre dans la commune.

L'accès à l'engrais minéral (ACCINT) : L'accès à l'engrais constitue la base dans l'utilisation de la pratique de fumure minérale. Toutefois, les problèmes d'impayés et beaucoup d'autres contraintes socio- 
culturelles constituent un frein au succès de ce processus.

L'appartenance à un groupement (APPGROP) : Plus le producteur n'appartient à un groupement, plus il a tendance à adopter cette stratégie. Donc, dans la commune de Ouaké, l'appartenance à un groupement favorise la pratique de la fumure minérale.

\section{Déterminants de l'adoption de la pratique d'agroforesterie}

Les résultats du modèle d'estimation des facteurs d'adoption de la pratique d'agroforesterie montrent que les variables, nombre d'actifs agricoles et appartenance à un groupement sont significatives au seuil de $10 \%$; la taille du ménage est significative au seuil de $5 \%$ et le mode de faire-valoir des terres mises en culture sont significatifs au seuil de $1 \%$. Elles ont une influence sur l'utilisation de la pratique d'agroforesterie en matière de gestion de la fertilité des sols.

La taille du ménage: elle a une influence négative sur la probabilité du choix de la stratégie d'agroforesterie comme pratique de gestion de la fertilité des sols. Cependant, il est à relever qu'avec le signe négatif du coefficient, ce résultat nous indique que plus le ménage est grand, moins il aura tendance à adopter cette stratégie face à la baisse de la fertilité.

L'appartenance à un groupement (APPGROP) : cette variable influence négativement l'adoption de la pratique d'agroforesterie par le producteur. Ainsi, plus le producteur appartient à un groupement ou à une taille du ménage élevée, moins il envisage l'utilisation de la pratique d'agroforesterie pour la gestion de la fertilité des sols.

Le nombre d'actifs agricoles (NACT): le nombre d'actifs agricoles a une influence positive sur l'adoption de l'agroforesterie. Cela suppose que, plus la main-d'œuvre familiale est disponible dans la zone, plus la probabilité de choisir l'agroforesterie est élevée.

Le mode de faire-valoir $(M V F)$ : la variable influence positivement la pratique d'agroforesterie. Seuls les producteurs ayant hérités les terres sont habileté à planter des arbres sur la parcelle en culture. Les autres, l'ayant acquis par don, seront vus comme s'accaparant de ces terres lorsqu'ils mèneront cette action.

\section{Déterminants de l'adoption de la pratique de lutte antiérosive}

L'analyse du Tableau 5 présentant les résultats du modèle d'estimation des facteurs d'adoption de la lutte antiérosive montre que parmi les variables introduites dans le modèle, le sexe, l'âge, l'accès à l'engrais minéral, le statut social, l'appartenance à un groupement, le niveau d'instruction du producteur et la taille du ménage ont une incidence sur l'utilisation de la lutte antiérosive dans la conservation des sols de la commune de Ouaké.

Le sexe du producteur (SEX) est significatif au seuil de 5\% et influence négativement l'utilisation de la lutte antiérosive comme méthode de conservation des sols. Cela suppose que les hommes utilisent plus la pratique de lutte antiérosive que les femmes

L'âge (AGE) a un impact significatif et négatif au seuil de $10 \%$ sur l'adoption de la lutte antiérosive. Plus le producteur est âgé, moins il songe à l'utilisation de la pratique de lutte antiérosive comme stratégie de conservation des sols. Les jeunes sont donc plus disposés à l'utilisation de cette pratique que les personnes âgées.

L'appartenance à un groupement $(A P P G R O P)$ : est significative au seuil de $10 \%$ et influence négativement la probabilité que le producteur adopte la lutte antiérosive comme pratique de gestion et de conservation des sols. Ce résultat admet aussi que, plus le producteur appartient au groupement, moins il opte pour l'utilisation de la pratique de lutte antiérosive.

L'accès à l'engrais minéral (ACCINT) influence négativement cette pratique et est significatif au seuil de $10 \%$. Les producteurs ayant accès à l'engrais minéral préfèrent ne pas adopter la lutte antiérosive pour autre pratique (fumure minérale). 
Le niveau d'instruction (EDUC): il est significatif au seuil de $5 \%$ et détermine positivement l'adoption de la pratique de lutte antiérosive. Une meilleure compréhension et l'esprit d'ouverture des lettrés aux innovations expliquent le fait que les producteurs instruits adoptent plus cette pratique introduite par les projets et programmes de protection de l'environnement que les producteurs non instruits.

La taille du ménage (TAILM): elle a un impact positif et significatif au seuil de $10 \%$ sur la pratique antiérosive. Les producteurs ayant une grande taille de ménage seront plus disposés à l'adoption de cette pratique.

Le statut social (STAT): influence aussi la décision de l'exploitant à adopter cette pratique. Plus le producteur possède un statut social élevé donc un niveau d'aisance élevé, plus il opte pour l'utilisation des innovations.

\section{Déterminants de la pratique de rotation et d'association de cultures appropriées}

La rotation et l'association de cultures appropriées sont une pratique traditionnelle, endogène à la population. L'âge, le nombre d'actifs agricoles sont significatifs au seuil de $10 \%$ et le niveau d'instruction au seuil de 5\%, sont les facteurs qui déterminent son adoption (Tableau 6) :

L'âge du producteur (AGE) a une incidence positive sur l'adoption de la pratique de rotation ou d'association de cultures appropriées. Plus le producteur est âgé, plus il opte pour l'utilisation de cette pratique. Ceci explique bien que c'est une pratique traditionnelle, mieux maîtrisée par les personnes âgées. Ces derniers sont très conservateurs et réticents envers les nouvelles technologies.

Le niveau d'instruction (EDUC): Le niveau d'éducation, quant à lui, influence négativement le choix de cette stratégie au seuil de $10 \%$. Plus le niveau des agriculteurs est élevé, moins ils auront tendance à adopter cette stratégie d'adaptation face à la baisse de la fertilité.

Le nombre d'actif agricole (NACT) influence négativement la pratique de l'association rotation. Plus un ménage a un nombre d'actifs agricoles élevé, moins il est réceptif à l'utilisation de cette pratique.

Tableau 1 : Noms, codes, modalités et signes attendus des coefficients des variables explicatives incluses dans le modèle de régression logit.

\begin{tabular}{|c|c|c|c|}
\hline Noms des variables & Code & Modalités & Signes attendus \\
\hline Age & AGE & - & $+/-$ \\
\hline Niveau d'instruction & EDUC & $\begin{array}{l}0=\text { pas allé à l'école } \\
1=\text { allé à l'école }\end{array}$ & + \\
\hline Taille du ménage & TAILM & & + \\
\hline Appartenance à un groupement & APPGROP & $\begin{array}{l}0=\text { n'appartient pas } \\
1=\text { appartient }\end{array}$ & $+/-$ \\
\hline Nombre d'actifs agricoles & NACT & - & + \\
\hline Sexe & SEX & $\begin{array}{l}0=\text { femme } \\
1=\text { homme }\end{array}$ & $+/-$ \\
\hline Accès à l'engrais minéral & ACCINT & $\begin{array}{l}0=\text { pas d'accès } \\
1=\text { accès }\end{array}$ & + \\
\hline Statut social & STAT & $\begin{array}{l}0=\text { faible } \\
1=\text { moyen } \\
3=\text { =élevé }\end{array}$ & + \\
\hline Contact avec un encadreur & CONTACT & $\begin{array}{l}0=\text { pas de contact } \\
1=\text { contact }\end{array}$ & + \\
\hline Mode de faire-valoir & MFV & $\begin{array}{l}0=\text { don } \\
1=\text { héritage }\end{array}$ & + \\
\hline
\end{tabular}


Tableau 2 : Superficies moyennes emblavées (en ha) par les enquêtés bénéficiant des différents pratiques de gestion de la fertilité des sols.

\begin{tabular}{|c|c|c|c|c|c|c|c|c|c|c|c|c|}
\hline \multirow[b]{2}{*}{ Villages } & \multicolumn{3}{|c|}{ Fumure minerale } & \multicolumn{3}{|c|}{ Agroforestérie } & \multicolumn{3}{|c|}{ Lutte antiérosive } & \multicolumn{3}{|c|}{ Rotation et association de cultures appropriées } \\
\hline & Maïs & Arachide & Riz & Maïs & Arachide & Riz & Maïs & Arachide & Riz & Maïs & Arachide & Riz \\
\hline Tchaladè & 0,44 & 0,15 & 0,19 & 0,03 & 0,1062 & 0,0031 & 0,02 & 0,025 & 0,0625 & 0,187 & 0,131 & 0,084 \\
\hline Assodè & 0,006 & 0 & 0 & 0,24 & 0,1656 & 0,05 & 0,087 & 0,031 & 0,2718 & 0,28 & 0,225 & 0,04375 \\
\hline Alayomdè & 0,27 & 0,031 & 0,0031 & 0,168 & 0,191 & 0,0125 & 0,056 & 0,056 & 0,278 & 0,128 & 0,203 & 0,0375 \\
\hline Total & & 0,3041 & & & $\mathbf{0 , 3 7 1 5}$ & & & 0,39 & & & & 0,3685 \\
\hline
\end{tabular}

Tableau 3 : Signification des modèles d'estimation des facteurs d'adoption.

\begin{tabular}{lcccc}
\hline & Fumure minérale & Agroforesterie & Lutte antiérosive & Rotation et association de cultures appropriées \\
\hline Nombre d'observation & 120 & 120 & 120 & 120 \\
Log pseudo likelihood & 130,936 & 125,783 & 119,390 & 142,291 \\
Pseudo R & $0,340 * * *$ & $0,40 * * *$ & $0,44 * * *$ & $0,193 * *$ \\
Probabilité de signification & 0,000 & 0,000 & 0,000 & 0,049 \\
Pouvoir de prédiction correcte & $71 \%$ & $72 \%$ & $79 \%$ & $65 \%$ \\
\hline
\end{tabular}

Tableau 4 : Résultat du modèle d'estimation des facteurs d'adoption de la fumure minérale.

\begin{tabular}{lccc}
\hline Variables & Coefficient & Erreur standard & Signification \\
\hline SEX & $2,034^{*}$ & 1,198 & 0,090 \\
AGE & 0,005 & 0,026 & 0,838 \\
EDUC & 0,493 & 0,497 & 0,321 \\
NACT & 0,155 & 0,104 & 0,135 \\
STAT & $-0,104$ & 0,311 & 0,738 \\
APPGROP & $0,967 *$ & 0,540 & 0,074 \\
CONTACT & $-0,383$ & 0,620 & 0,537 \\
ACCINT & $1,407 * *$ & 0,586 & 0,016 \\
MFV & $1,076^{* *}$ & 0,522 & 0,039 \\
Pouvoir de prédiction correcte : $70,80 \%$. Les variables marquées * sont significatives à $10 \%$ et ** significatives à $5 \%$.
\end{tabular}


J. A. YABI et al. / Int. J. Biol. Chem. Sci. 10(2): 779-792, 2016

Tableau 5 : Résultat du modèle d'estimation des facteurs d'adoption de l'agroforesterie.

\begin{tabular}{lccc}
\hline Variables & Coefficient & Erreur standard & Signification \\
\hline SEX & $-21,040$ & 11240 & 0,999 \\
AGE & 0,038 & 0,028 & 0,177 \\
NACT & $0,242^{*}$ & 0,078 & 0,012 \\
APPGROP & $-0,983^{*}$ & 0,146 & 0,062 \\
STAT & 0,158 & 0,303 & 0,601 \\
TAILM & $-0,196^{* *}$ & 0,592 & 0,073 \\
CONTACT & $-0,528$ & 0,656 & 0,421 \\
ACCINT & 0,416 & 0,618 & 0,501 \\
MFV & $2,080^{* * *}$ & 0,613 & 0,001 \\
EDUC & 0,111 & 0,475 & 0,815 \\
\hline correcte : $72,50 \%$. Les variables marquées * sont significatives à $10 \% ; * *$ significatives à $5 \%$ et *** significatives à $1 \%$.
\end{tabular}

Tableau 6 : Résultat du modèle d'estimation des facteurs d'adoption de la lutte antiérosive.

\begin{tabular}{lccc}
\hline Variables & Coefficient & Erreur-type & Signification \\
\hline SEX & $-2,253^{* *}$ & 1,190 & 0,048 \\
AGE & $-0,056^{* *}$ & 0,029 & 0,050 \\
ACCINT & $-1,328^{* *}$ & 0,516 & 0,076 \\
STAT & $0,663^{* *}$ & 0,320 & 0,038 \\
APPGROP & $-0,946^{*}$ & 0,559 & 0,091 \\
CONTACT & 0,357 & 0,661 & 0,589 \\
EDUC & $0,915^{*}$ & 0,617 & 0,031 \\
MFV & $-0,598$ & 0,535 & 0,264 \\
TAILM & $0,088^{*}$ & 0,053 & 0,096
\end{tabular}

Pouvoir de prédiction correcte : $79,20 \%$. Les variables marquées * sont significatives à $10 \%$ et ** significatives à $5 \%$. 
Tableau 7 : Résultat du modèle d'estimation des facteurs d'adoption de la rotation et l'association de cultures appropriées.

\begin{tabular}{lccc}
\hline Variables & Coefficient & Erreur standard & Signification \\
\hline SEX & $-0,005$ & 0,025 & 0,830 \\
AGE & $1,553^{*}$ & 0,930 & 0,095 \\
TAILM & 0,100 & 0,065 & 0,128 \\
NACT & $-0,238^{*}$ & 0,134 & 0,077 \\
STAT & $-0,336$ & 0,300 & 0,262 \\
APPGROP & 0,472 & 0,537 & 0,379 \\
CONTACT & 0,586 & 0,576 & 0,309 \\
ACCINT & 0,220 & 0,569 & 0,699 \\
MFV & $-0,692$ & 0,501 & 0,167 \\
EDUC & $-0,927 * *$ & 0,485 & 0,05 \\
Pouvoir de prédiction correcte $: 65 \%$. Les variables marquées * sont significatives à $10 \%$ et ** significatives à 5\%
\end{tabular}

\section{DISCUSSION}

Plusieurs auteurs sont parvenus à la conclusion que les facteurs déterminant l'adoption d'une innovation introduite en milieu rural sont liés non seulement à la technologie mais aussi aux conditions socioéconomiques et culturelles du producteur. Toutefois, le sens de cette influence varie d'une thématique à une autre et selon le contexte. Ainsi, des résultats de cette étude, la taille du ménage a une influence négative sur l'adoption de l'agroforesterie. La taille du ménage constitue une variable clé dans l'adoption de nouvelles technologies. Ce résultat corrobore les conclusions de Folefack et al. (2012) qui ont trouvé que la taille du ménage a eu un effet significatif et négatif sur la probabilité du choix de la stratégie de fumure organique. En effet, l'accès à l'engrais par le producteur a un effet positif et significatif sur l'adoption de la fumure minérale. Ce résultat est semblable aux résultats obtenus par Bello et al. (2012) qui ont trouvé que les quantités d'engrais qu'achètent les producteurs et les doses qu'ils appliquent dépendent de la disponibilité de ces engrais dans les circuits de distribution et d'approvisionnement.

La pratique de lutte antiérosive constitue une innovation dont l'utilisation par le producteur engendre des risques auxquels les personnes âgées ne sont pas prédisposées. Les résultats de cette étude viennent ainsi confirmer ceux de Zegeye et al. (2001) et de Ouédraogo (2003) qui ont abouti à la conclusion que l'adoption de nouvelles technologies exige un certain niveau de risque associé à la décision du choix des innovations. De même, les jeunes producteurs moins expérimentés sont enclins à prendre plus de risque que les producteurs plus âgés mais ce résultat infirme ceux de Adégbola et Adékambi (2008); Sall et al. (2000); Chanou (2007) et Dakin (2008) qui ont montré que les producteurs les plus âgés (plus expérimentés) adopteraient plus facilement les innovations que les jeunes.

Selon Azontondé (2004), l'éducation est un facteur affectant l'adoption et l'application des innovations technologiques en milieu rural. En effet, plus les producteurs sont instruits, plus ils sont ouverts aux innovations technologiques. Le niveau d'instruction accroît donc le sens de l'innovation, l'habileté et la facilité d'apprécier les nouvelles technologies (Dakin, 2008). Une meilleure compréhension et l'esprit d'ouverture des lettrés aux innovations expliquent également nos résultats qui confirment ceux de Houeyissan (2006) sur les déterminants du choix et la volonté des riziculteurs du département des 
Collines à payer les semences des variétés améliorées mais ne corroborent pas ceux de Dakin (2008) qui est arrivé à la conclusion suivant laquelle le niveau d'instruction affecte négativement l'adoption de des technologies vulgarisées par le PDRT.

Le sens de variation des déterminants de l'adoption d'une innovation introduite en milieu rural dépend donc du contexte de l'étude et des suppositions faites par l'auteur au cours de ses analyses. Toutefois, il faut remarquer que la plupart des résultats de cette étude corroborent ceux des travaux antérieurs effectués sur les déterminants des innovations introduites en milieu rural.

\section{Conclusion}

Les facteurs déterminants l'adoption des pratiques culturales de gestion de la fertilité des sols sont variables d'une pratique à une autre. La plupart de ces facteurs sont socio-économiques et techniques et sont non seulement liée aux producteurs mais aussi à la pratique. L'amélioration des facteurs déterminants l'adoption de chaque pratique et la mise en place d'une politique d'amélioration de la connaissance des producteurs de la performance de ces pratiques pourraient, dans une certaine mesure, faciliter leur adoption et une large diffusion de ces dernières dans la commune de Ouaké pour une meilleure gestion de la fertilité des sols.

\section{CONFLIT D'INTERETS}

Les auteurs déclarent qu'il n'y a aucun conflit d'intérêt.

\section{CONTRIBUTIONS DES AUTEURS}

JAY a participé à la correction du manuscrit; IAL a participé à l'analyse de données, la rédaction et la correction du manuscrit; FXB: a participé à l'analyse de données et a participé à la production vegetale et à la science du sol; RLA a collecté les données et a élaboré le projet d'article ; CAO a collecté les données.

\section{REMERCIEMENTS}

L'équipe de chercheurs adresse ses remerciements aux autorités de la mairie de Ouaké, au Responsable au Développement Rural du Secteur Communal du Développement Agricole. L'équipe remercie aussi les agents enquêteurs pour la qualité de données collectées.

\section{REFERENCES}

Adégbola P, Adékambi SA. 2008. Taux et déterminants de l'adoption des variétés améliorées d'igname développées par l'IITA. Rapport d'étape, 31p.

Adésina A, Mbila D, Nkamleu G, Endamana D. 2000. Econometric analysis of the determinants of adoption of alley farming by farmers in the forest zone of southwest Cameroon. Agricultural Economics, 80: 255-265.

Azontondé R. 2004. Impact économique de l'adoption des pratiques de la gestion intégrée de la fertilité des sols (GIFS) au Sud-Bénin: Cas d'Aplahoué (commune de Klouekanme) et de Banigbé (commune d'Ifangni). Thèse d'ingénieur agronome, Faculté des Sciences Agronomiques, Université d'Abomey- Calavi, Bénin, p.154.

Batamoussi Hermann M, Moumouni I, Tokoro Orou Méré SBJ. 2015. Contribution à l'amélioration des pratiques paysannes de production durable de coton (Gossypium hirsutum) au Bénin : cas de la commune de Banikoara. Int. J. Biol. Chem. Sci., 9(5): 2401-2413. DOI : http://dx.doi.org/10.4314/ijbcs.v9i5.12

Bagi FS. 1983. A logit Model of farmers' adoption decisions about credit. Southern J. Agric. Econ. 15: 32-76.

Bello S, Ahanchede A, Amadji G, Gbehounou G, Aho N. 2012. Effet de la fumure minérale sur l'enherbement et la production de l'oignon (Allium 
cepa L.) au Nord-Est du Bénin. Int. J. Biol. Chem. Sci. 6(6): 4058-4070, http://ajol.info/index.php/ijbcs

Chanou P. 2007. Efficacité économique de la gestion des bas-fonds rizicoles de Gogounou dans une perspective d'accroissement de leurs performances. Thèse d'ingénieur agronome, Faculté d'Agronomie, Université de Parakou, Bénin, p. 74.

CIMMYT, ECONOMICS PROGRAM. 1993. The adoption of agricultural technology: A guide for survey design. Mexico, D.F.: CIMMYT. 88 pp

Cramer JS. 1991. The Logit Model for Economists. London and New York: Edward Arnold; 154 pp

Dakin L. 2008. Impact socio-économique des interventions du PDRT sur les femmes rurales du département de la Donga. Thèse d'ingénieur agronome, Faculté d'Agronomie Université de Parakou, Bénin, p.106.

De Janyry A. 1994. Farm Non-farm synergies in Africa: Discussion. American Journal of Agricultural Economics, 76(2): 1183-1185.

Falusi AO. 1975. Application of MultiVariate Probit To Fertilizer Use Decision: Sample Survey of Farmers In Three States In Nigeria. J. Rural Econ. Development, 9(1): 49-66.

Feder G. 1982. Adoption of interrelated agricultural innovations: complementary and the risk, scale and credit. American Journal of Agricultural Economics, 12: 59-73.

Folefack DP, Sale A, Wakponou A. 2012. Facteurs affectant l'utilisation de la fumure organique dans les exploitations agricoles en zone sahélienne du Cameroun. Afrique Science: Revue Internationale des Sciences et Technologie, 8(2): 22-33.

Houeyissan S. 2006. Déterminants des choix et de la volonté de payer les semences des variétés améliorées de riz dans le
Département des Collines, Thèse d'ingénieur agronome, Faculté des Sciences Agronomiques, Université d'Abomey- Calavi, Bénin, P.94

Hurlin C. 2003. Econométrie des variables qualitatives. Cours de maîtrise d'économétrie, France Université d'Orléans, 59 p.

Jama B, Gonzalo P. 2008. Agriculture in Africa: Strategies to Improve and Sustain Smallholder Production Systems. Ann. N.Y. Acad. Sci. New York 1136: 218-232

Kebede Y, Gunjal K, Coffin G. 1990. Adoption of new technologies in Ethiopian Agriculture: The case of Tegulet-Bulga District, Shoa Province. Agricultural Economics, 4(1): Elsevier Science Publishers, Amsterdam

Maddala GS. 1983. Limited Dependent and Qualitative variables. Econometrics Cambridge University Press: London, pp-67.

Mbetid-Bessane E. 2014. Adoption et intensification du Nouveau Riz pour l'Afrique en Centrafrique. Tropicultura, 32(1) :16-21.

Nouatin G. 2008. Diagnostics de quelques pratiques de gestion et de conservation de la fertilité des terres dans la commune de Ouaké. Etude exploratoire. Rapport technique. Faculté d'Agronomie, Université de Parakou. Parakou, Bénin, p.64.

Ouédraogo R. 2003. Adoption et intensité d'utilisation de la culture attelée, des engrais et des semences améliorées dans le centre nord du Burkina. CEDRES, Université de Ouagadougou, Burkina Faso, p.107.

Ouédraogo M, Dembélé Y, Somé L. 2010. Perceptions et stratégies d'adaptation aux changements des précipitations : cas des paysans du Burkina Faso. Sécheresse, 21(2): 87-96.

Renard JF, Cheikh L, Knips V. 2004. L'élevage et l'intégration régionale en 
Afrique de l'Ouest, Ministère des Affaires étrangères - FAO-CIRAD, 37 p.

Rogers EM. 1983. Diffusion of Innovations. ( $3^{\text {rd }}$ edn). The Free Press: New York; $90 \mathrm{p}$.

Sale A, Folefack PD, Obwoyere GO, Lenah Wati N, Lendzemo WV, Wakponou A. 2014. Changements climatiques et déterminants d'adoption de la fumure organique dans la région semi-aride de Kibwezi au Kenya. Int. J. Biol. Chem. Sci. 8(2): 680-694. DOI : http://dx.doi.org/10.4314/ijbcs.v8i2.24

Sall S, Norman D, Featherstone A. 2000. Quantitative assessment of improved rice variety adoption: the farmer's perspective. Agricultural Systems, 66: 129-144.
Sermé I, Outtara K, Logah V, Taounda JB, Pale S, Quansah C, Abaidoo R. 2015. Impact of tillage and fertility management options on selected soil physical properties and sorghum yield. Int. J. Biol. Chem. Sci., 9(3): 11541170 . DOI http://dx.doi.org/10.4314/ijbcs.v9i3.2

Van Den Ban AW, HAwkins HS, Brouwers J, Boob CA. 1994. La vulgarisation Rurale en Afrique; Edition Karthala et CTA ; 115-144.

Zégeyé T, Tadesse B, Tesfaye S. 2001. Determinants of adoption of improved maize technologies in major maize growing regions of Ethiopia. Second National Maize Workshop of Ethiopia, Ethiopia, 12-16. 